\title{
Synthesis, Spectrometric Characterization, X-Ray Crystallography and Quantum Chemical Calculations of 2-oxo-2H-chromen-7-yl Propionate
}

\author{
Akoun Abou ${ }^{1, *}$, Abdoulaye Djandé2, Bintou Sessouma ${ }^{2}$, Rita Kakou Yao ${ }^{3}$, Olivier Ouari ${ }^{4}$, \\ Adama Saba ${ }^{2}$ \\ ${ }^{1}$ Department of Training and Research in Electrical and Electronic Engineering, Research Team: Instrumentation, Image and Spectroscopy, \\ Félix Houphouët-Boigny National Polytechnic Institute, Yamoussoukro, Côte d'Ivoire \\ ${ }^{2}$ Department of Chemistry, Laboratory of Molecular Chemistry and Materials, University Joseph KI-ZERBO, Ouagadougou, Burkina Faso \\ ${ }^{3}$ Department of Physics, Laboratory of Crystallography and Molecular Physics, University Félix Houphouët-Boigny, Abidjan, Côte d'Ivoire \\ ${ }^{4}$ Department of Chemistry, Radical Chemistry Institute, Aix-Marseille University, Marseille, France
}

Email address:

abouakoun@gmail.com (A. Abou)

${ }^{*}$ Corresponding author

\section{To cite this article:}

Akoun Abou, Abdoulaye Djandé, Bintou Sessouma, Rita Kakou Yao, Olivier Ouari, Adama Saba. Synthesis, Spectrometric Characterization, X-Ray Crystallography and Quantum Chemical Calculations of 2-oxo-2H-chromen-7-yl Propionate. American Journal of Heterocyclic Chemistry. Vol. 5, No. 2, 2019, pp. 37-48. doi: 10.11648/j.ajhc.20190502.13

Received: June 17, 2019; Accepted: July 12, 2019; Published: July 24, 2019

\begin{abstract}
The title compound, (I), has been solved by direct methods and refined to a final $\mathrm{R}$ value of 0.038 for 1835 independent reflections. In the structure, the planar [r.m.s deviation $=0.014 \AA$ ] chromen-2-one ring and the 7-propionate side chain are inclined to one another at an acute angle of $65.34(9)^{\circ}$. The molecules form $\mathrm{R}_{4}{ }^{4}(30)$ tetrameric units via $\mathrm{C}-\mathrm{H} \cdots \mathrm{O}$ interactions which extend into layers approximately parallel to the $a b$ plane. Furthermore, the crystal structure is supported by $\pi-\pi$ stacking interactions between neighbouring benzene and pyrone or coumarin rings [centroid-centroid distances in the range 3.6097(8)-3.6475(9) $\AA$ ], as well as $\mathrm{C}-\mathrm{H} \cdots \pi$ interactions $[\mathrm{H} \cdots$ centroid distances in the range $2.95-3.00 \AA]$. The molecular geometry of (I) was also optimized using density functional theory (DFT/RB3LYP), RMP2 and RHF methods with the 6$311^{++} \mathrm{G}(d, p)$ basis set in ground state. The theoretical data resulting from these quantum chemical calculations are in good agreement with the observed structure, although the observed $\mathrm{C}-\mathrm{O}-\mathrm{C}-\mathrm{C}$ torsion angle between the coumarin ring system and the 7-propionate side chain $\left(121.49(16)^{\circ}\right)$ is somewhat lower than the DFT/RB3LYP calculated value $\left(132.32^{\circ}\right)$ and larger than the RMP2 $\left(114.65^{\circ}\right)$ and the RHF $\left(69.19^{\circ}\right)$ values. Hirshfeld surface analysis has been used to confirm and quantify the supramolecular interactions.
\end{abstract}

Keywords: Coumarin Ester, Hirshfeld Surface Analysis, Quantum Chemical Calculations

\section{Introduction}

Coumarins and their derivatives constitute one of the major classes of naturally occurring compounds and interest in their chemistry continues unabated because of their usefulness as biologically active agents. They also form the core of several molecules of pharmaceutical importance. Coumarin and its derivatives have been reported to serve as anti-bacterial [1], anti-oxidant [2] and anti-inflammatory agents [3].

\section{Experimental and Theoretical Methods}

\subsection{Synthesis}

To a solution of propionic anhydride $(6.17 \mathrm{mmol} ; 0.85 \mathrm{ml})$ in dried diethyl ether $(25 \mathrm{ml})$, was added dried pyridine (4.7 molar equivalents; $2.35 \mathrm{ml})$ and 7-hydroxycoumarin $(6.17$ $\mathrm{mmol} ; 1 \mathrm{~g})$ in small portions over $30 \mathrm{~min}$. The mixture was 
then left under agitation at room temperature for 3 hours and poured into $40 \mathrm{ml}$ of chloroform. The solution was acidified with diluted hydrochloric acid until the $\mathrm{pH}$ was 23. The organic layer was extracted, washed with water to neutrality, dried over $\mathrm{MgSO}_{4}$ and the solvent removed. The crude product was washed with petroleum ether and recrystallized from acetone. White crystals of the title compound were obtained (yield 89.5\%); M.p: 366-368 K.

\subsection{Electrospray Ionisation Mass Spectrum}

Mass spectrometry is a highly valuable technique in the field of structural biochemistry. Electrospray ionization mass spectrometry (ESI-MS), with an accuracy of about $0.01 \%$, provides an extremely sensitive method for determining the precise molecular mass of small and biological molecules. The spectrum of figure 1 was recorded on a 3200 QTRAP (Applied Biosystems SCIEX) spectrometer equipped with a pneumatically assisted air pressure ionization (API) source for $\mathrm{ESI}^{-\mathrm{MS}^{+}}$experiment.

\section{3. ${ }^{1} \mathrm{H}$ and ${ }^{13} \mathrm{C}$ Spectra}

${ }^{1} \mathrm{H}$ and ${ }^{13} \mathrm{C}$ NMR spectra figures 2 and 3 were recorded on a Bruker TopSpin spectrometer at 400 and $100 \mathrm{MHz}$, respectively, using $\mathrm{CDCl}_{3}$ as internal standard (chemical shifts in $\delta$ values, $\mathrm{J}$ in $\mathrm{Hz}$ ).

\subsection{Crystal Structure Analysis}

Diffraction data for the title compound was collected on Rigaku Oxford Diffraction SuperNova, Dual, $\mathrm{Cu}$ at zero, AtlasS2 diffractometer [6] using a mirror monochromator and $\mathrm{Cu} K \alpha$ radiation $(\lambda=1.54184 \AA)$ at $298 \mathrm{~K}$. The structure was solved by direct methods using SIR2014 [7] and implemented in the WinGX [8] program suite. The refinement was carried out by full-matrix least squares method on the positional and anisotropic temperature parameters of the non-hydrogen atoms, or equivalently corresponding to 146 crystallographic parameters, using SHELXL2014 program [9]. All $\mathrm{H}$ atoms were placed in calculated positions $[\mathrm{C}-\mathrm{H}=0.93$ (aromatic), 0.96 (methyl) or $0.97 \AA$ (methylene group)] and refined using a riding model approximation with $\operatorname{Uiso}(\mathrm{H})$ constrained to 1.2 (aromatic and methylene group) or 1.5 (methyl group) times Ueq of the respective parent atom. Data collection is by CrysAlis PRO [6], cell refinement by CrysAlis PRO [6], and data reduction by CrysAlis PRO [6]. The general-purpose crystallographic tool PLATON [10] was used for the structure analysis and presentation of the results. Details of the data collection conditions and the parameters of the refinement process are given in Table 1.

CCDC-1845532 contains the supplementary crystallographic data for this paper. These data can be obtained free of charge from The Cambridge Crystallographic Data Centre via www.ccdc.cam.ac.uk/data_request/cif; e-mail: deposit@ccdc.cam.ac.uk.

\subsection{Hirshfeld Surface}

Molecular Hirshfeld surfaces of 2-oxo-2H-chromen-7-yl propionate were calculated using a standard (high) surface resolution, and with the three-dimensional $d_{\text {norm }}$ surfaces mapped over a fixed colour scale of -0.205 (red) to 1.418 a.u. (blue) with the program CrystalExplorer 3.1 [11].

\subsection{Computational Procedures}

The geometry optimization of compound (I) was performed by using the density functional theory (DFT) with restricted B3LYP exchange correlation functional, restricted Moller-Plesset perturbation theory (RMP2) and restricted Hartree-Fock (RHF) methods with a $6-311^{++} \mathrm{G}(\mathrm{d}, \mathrm{p})$ basis set. The crystal structure in the solid state was used as the starting structure for the calculations. All calculations are performed with the GAUSSIAN09 program package [12].

\section{Results and Discussions}

\subsection{Spectra Analysis}

\subsubsection{Interpretation of Electrospray Ionisation Mass Spectrum}

In the spectrum Figure 1, the exploitation of the peak positions of $[\mathrm{M}+\mathrm{H}]^{+},\left[\mathrm{M}+\mathrm{NH}_{4}\right]^{+}$and $[\mathrm{M}+\mathrm{Na}]^{+}$ions at $\mathrm{m} / \mathrm{z}$ ratio $=219.1,236.1$ and 241.1 respectively, afforded the unique molecular weight of $218.1 \mathrm{~g} \cdot \mathrm{mol}^{-1}$ which is consistent with the chemical formula $\mathrm{C}_{12} \mathrm{H}_{10} \mathrm{O}_{4}$.

\subsection{2. ${ }^{1} \mathrm{H}$ Spectrum Analysis}

The experimental values (chemical shifts and couplings) taken from the ${ }^{1} \mathrm{H}$ NMR spectrum Figure 2 show a triplet signal at $1.3 \mathrm{ppm}$ due to the methyl group protons $(\mathrm{t}, 3 \mathrm{H}, J=$ $8 \mathrm{~Hz}, \mathrm{H}-12)$. A quadruplet at $2.65 \mathrm{ppm}$ exhibits methylene protons (q, 2H, $J=8 \mathrm{~Hz}, \mathrm{H}-11)$. Further, the chemical shifts in the range 6-8.5 ppm with doublets as signals, correspond to aromatic protons: $7.7(\mathrm{~d}, 1 \mathrm{H}, J=9.5 \mathrm{~Hz}, \mathrm{H}-3), 7.5(\mathrm{~d}, 1 \mathrm{H}$, $J=8.4 \mathrm{~Hz}, \mathrm{H}-9), 7.12$ (d, 1H, $J=2.1 \mathrm{~Hz}, \mathrm{H}-6), 7.05$ (dd, 1H, $J=8.4,2.1 \mathrm{~Hz}, \mathrm{H}-8), 6.4(\mathrm{~d}, 1 \mathrm{H}, J=9.5 \mathrm{~Hz}, \mathrm{H}-2)$ Figure 4.

\subsection{3. ${ }^{13}$ C Spectrum Analysis}

The ${ }^{13} \mathrm{C}$ NMR spectrum Figure 3 exhibits, as expected, twelve peaks.

Chemical shift (ppm) and the corresponding carbon: 160.37 (C-1), 110.41 (C-2), 142.87 (C-3), 128.53 (C-9), 116.59 (C-8), 154.71 (C-7), 116.02 (C-6), 153.33 (C-5), 118.41 (C-4), 172.25 (C-10), 27.73 (C-11) and 8.91 (C-12) figure 4 .

\subsubsection{Conclusion}

The combination of the above results originating from the spectra analysis confirm the molecule drawn in Figure 4. Other studies such as X-ray and theoretical calculations have been used to confirm this conclusion. 
- +Q1: 3 MCA scans from Sample 1 (C2) of C2_d3aa_Q1_210_270_DP20.wiff (Turbo Spray)

$214.2 \bullet$

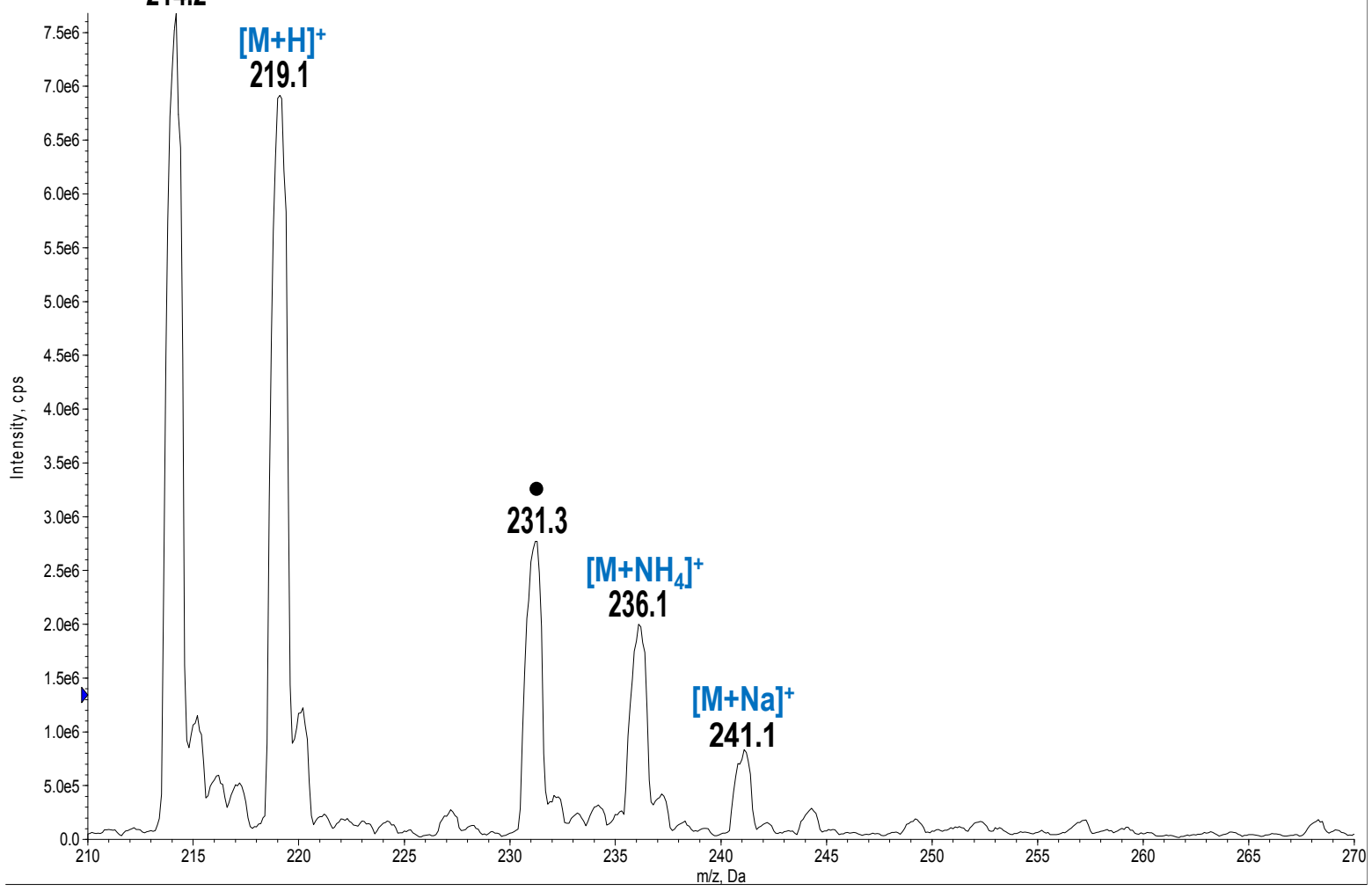

Figure 1. ESI-MS spectrum of compound I.

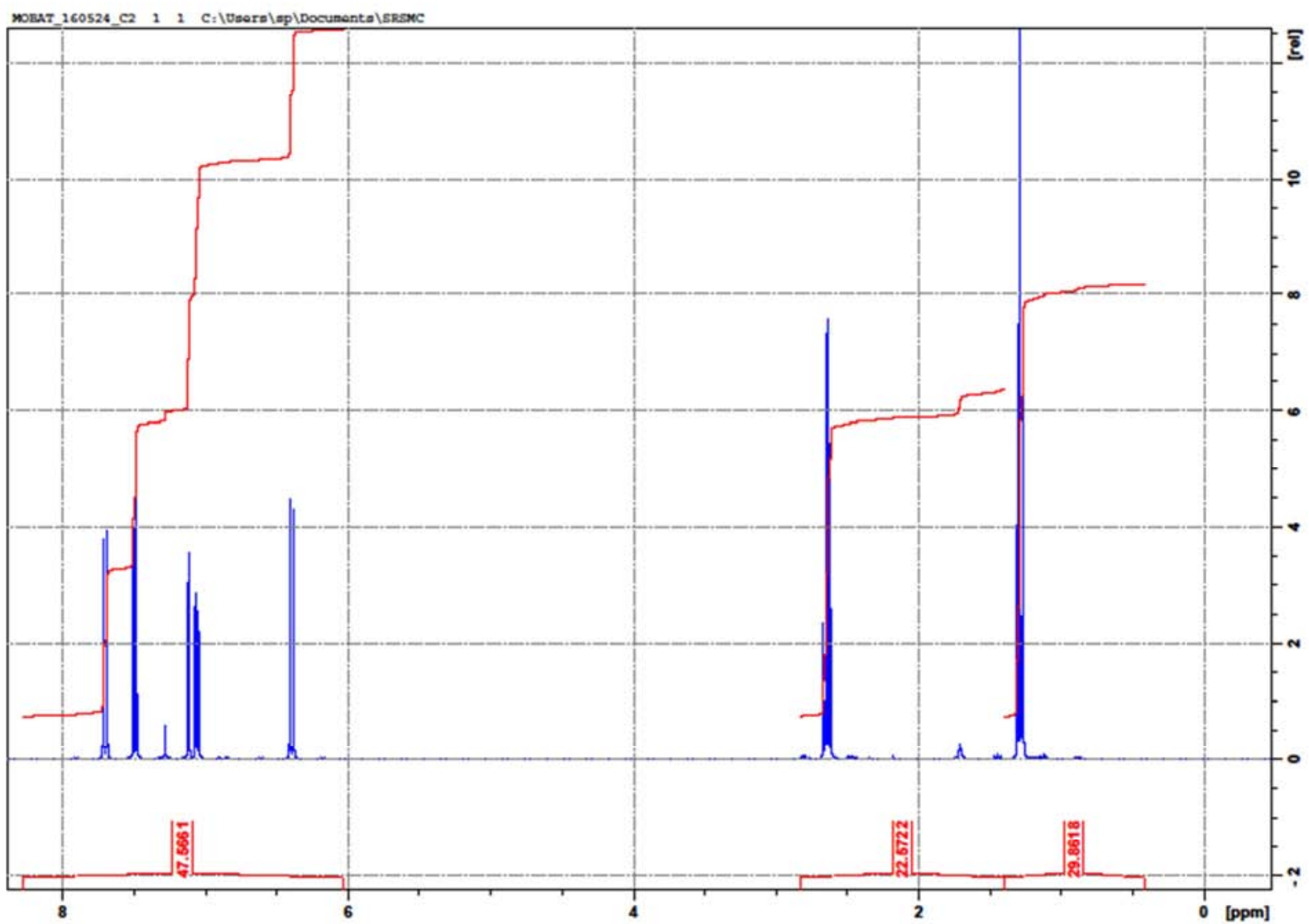

Figure 2. ${ }^{1} \mathrm{H} N \mathrm{NR}$ Spectrum of (I) in $\mathrm{CDCl}_{3}$. 


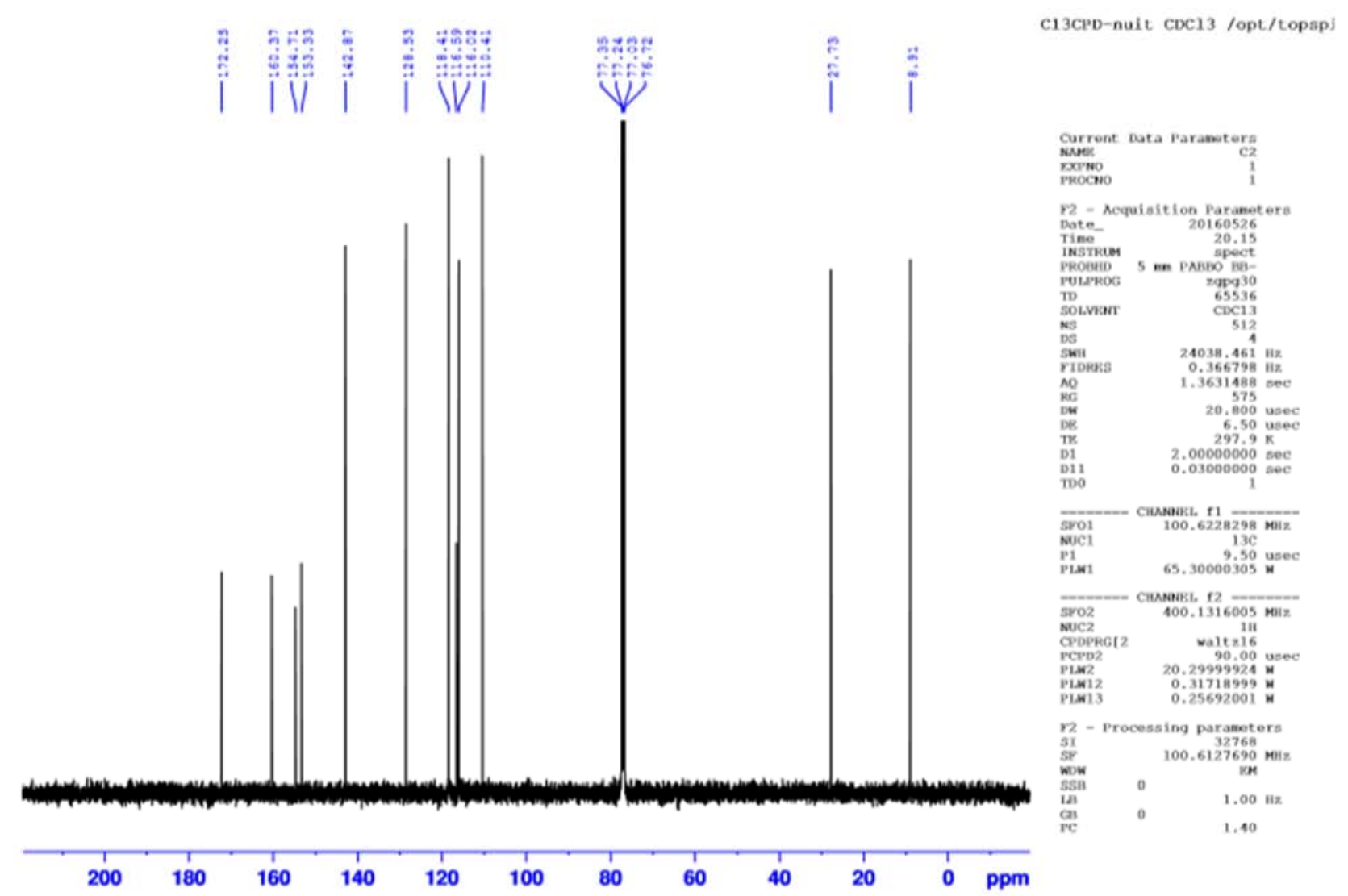

Figure 3. ${ }^{13} \mathrm{C}$ NMR Spectrum of (I) in $\mathrm{CDCl}_{3}$.

\subsection{Structural Description}

The molecular structure of the title coumarin derivative, (I), is illustrated in Figure 4.

\subsubsection{Geometry and Conformation}

The positional parameters, interatomic distances, bond angles and torsion angles are presented in Tables 4, 5, 6 and 7. The analysis and interpretation of the geometrical characteristics relating to bond distances compared to standard values [13] indicate that carbon-oxygen bonds: $[\mathrm{d}(\mathrm{C} 1-\mathrm{O} 1)=1.377(2) \AA$ and $\mathrm{d}(\mathrm{C} 5-\mathrm{O} 1)=1.3766(18) \AA]$ are characteristic of $\mathrm{Car}-\mathrm{O}(2)$ simple bond observed in other strctures in alkyl ethers [14-15]. Also, the distance d(C10$\mathrm{O} 4)=1.190(2) \AA$ and $\mathrm{d}(\mathrm{C} 10-\mathrm{O} 3)=1.361$ (2) $\AA$ indicate characteristic values of ester moiety. Furthermor, an inspection of the bond lengths of the coumarin ring shows that there is a slight asymmetry of the electronic distribution around the pyrone ring: the $\mathrm{C} 2-\mathrm{C} 3[1.334$ (3) $\AA]$ and $\mathrm{C} 1-$ C2 $\left[\begin{array}{lll}1.440 & (3) \AA]\end{array}\right.$ respectively, than those expected for a Car-Car bond. This suggests that the electron density is preferentially located in the $\mathrm{C} 2-\mathrm{C} 3$ bond of the pyrone ring, as seen in other coumarin derivatives [16-17].

In the crystal structure, The coumarin fragment is planar (r.m.s deviation $=0.014 \AA$ ) and oriented at an acute angle of $65.34(9)^{\circ}$ with respect to the planar propionate $\mathrm{O} 3 / \mathrm{O} 4 / \mathrm{C} 10$ $\mathrm{C} 12$ moiety (r.m.s deviation $=0.014 \AA$ ).

Table 1. Crystal data and details of the structure determination.

\begin{tabular}{|c|c|c|c|}
\hline chemical formula & $\mathrm{C}_{12} \mathrm{H}_{10} \mathrm{O}_{4}$ & Theta range for data collection $\left[{ }^{\circ}\right]$ & $6.0930-65.0690$ \\
\hline Formula weight & 218.20 & Crystal size $\left[\mathrm{mm}^{3}\right]$ & $0.24 \times 0.10 \times 0.03$ \\
\hline Temperature $[\mathrm{K}]$ & 298 & Index ranges & $-14 \leq \mathrm{h} \leq 10 ;-6 \leq \mathrm{k} \leq 7 ;-17 \leq \mathrm{l} \leq 17$ \\
\hline Wavelength $\lambda[\AA]$ & 1.54184 & Refections collected & 5891 \\
\hline Crystal system & Monoclinic & Absorption coefficient $\left[\mathrm{mm}^{-1}\right]$ & 0.858 \\
\hline Space group & $\mathrm{P} 2{ }_{1} / \mathrm{c}$ & Theta full $\left[{ }^{\circ}\right]$ & 65.360 \\
\hline Unit cell dimensions & & $\mathrm{F}(000)$ & 456 \\
\hline $\mathrm{a}[\AA]$ & $12.3296(4)$ & Refinement method & Full-matrix least squares on $\mathrm{F}^{2}$ \\
\hline $\mathrm{c}[\AA]$ & $14.7769(5)$ & Goodness of fit & 1.03 \\
\hline$\alpha\left[^{\circ}\right]$ & 90 & Final $\mathrm{R}$ indices $\left[\mathrm{F}^{2}>2.0 \sigma\left(\mathrm{F}^{2}\right)\right]$ & $\mathrm{R} 1=0.038, \mathrm{wR} 1=0.1071$ \\
\hline$\beta\left[{ }^{\circ}\right]$ & $101.404(4)$ & Density calculated $\left[\mathrm{g} \cdot \mathrm{cm}^{-3}\right]$ & 1.351 \\
\hline$\gamma\left[{ }^{\circ}\right]$ & 90 & Independent reflections & 1835 \\
\hline Volume $\left[\AA^{3}\right]$ & $107252(6)$ & $\Delta \rho_{\max }, \Delta \rho_{\min }\left(\mathrm{e} \AA^{-3}\right)$ & $0.19,-0.11$ \\
\hline
\end{tabular}




\begin{tabular}{llll}
\hline chemical formula & $\mathbf{C}_{\mathbf{1 2}} \mathbf{H}_{\mathbf{1 0}} \mathbf{O}_{\mathbf{4}}$ & Theta range for data collection [ ${ }^{\circ}$ ] & $\mathbf{6 . 0 9 3 0 - 6 5 . 0 6 9 0}$ \\
\hline $\mathrm{Z}$ & 4 & $\mathrm{R}$ indices (all data) & 0.0456 \\
$\begin{array}{l}\text { Crystal description- } \\
\text { crystal colour }\end{array}$ & $\begin{array}{l}\text { Prism } \\
\text { colorless }\end{array}$ & $\mathrm{R}_{\text {int }}$ & 0.026 \\
& & multi-scan; \\
& & & $\begin{array}{l}\text { CrysAlisPro 1.171.38.43 (Rigaku Oxford Diffraction, } \\
\text { Diffractometer }\end{array}$ \\
& $\begin{array}{l}\text { SuperNova, Dual, Cu } \\
\text { at zero, AtlasS2 }\end{array}$ & Absorption correction & $\begin{array}{l}\text { 2015) } \\
\text { Empirical absorption correction using spherical } \\
\end{array}$ \\
& & & $\begin{array}{l}\text { harmonics, implemented in SCALE3 ABSPACK scaling } \\
\text { algorithm. }\end{array}$ \\
\hline
\end{tabular}

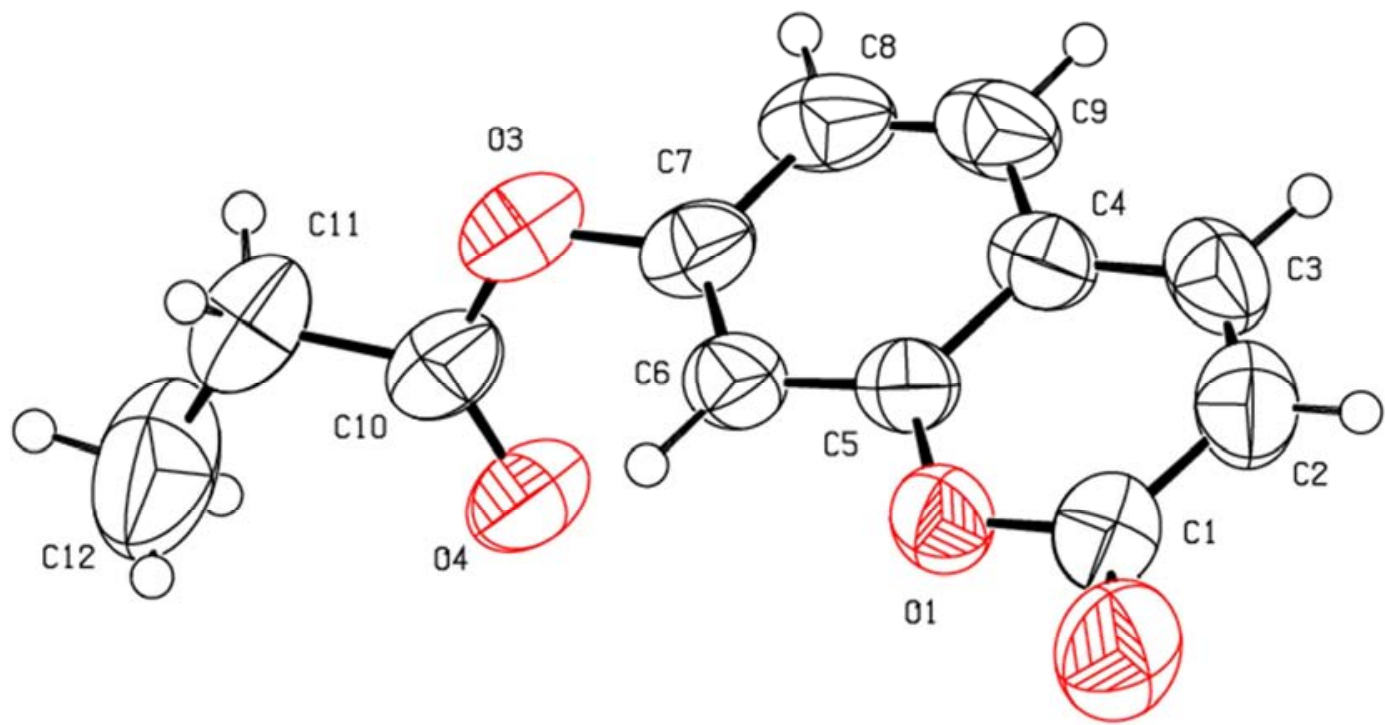

02

Figure 4. An ORTEP [8] view of the title complex with the atomic numbering scheme. Displacement ellipsoids are shown at the 50\% probability level.

\subsubsection{Supramolecular Features}

In the three-dimensional crystal packing, molecules form cyclic tetramers of $\mathrm{R}_{4}{ }^{4}(30)$ motifs [18] via two independent intermolecular $\mathrm{C} 8-\mathrm{H} 8 \ldots \mathrm{O} 4(\mathrm{x}, \mathrm{y}+1, \mathrm{z})$ and $\mathrm{C} 12-$ $\mathrm{H} 12 \mathrm{~B} \ldots \mathrm{O} 2(\mathrm{x}+1, \mathrm{y}, \mathrm{z})$ hydrogen bond interactions along the $a$ and $b$ axis. These tetramolecular aggregates extend into layers approximately parallel to the $a b$ plane Figure 5 . In addition, close contacts [H12A...C10(-x,-1/2+y,1/2-z) $=2.81$ $\AA],[\mathrm{H} 9 \ldots \mathrm{C} 4(1-\mathrm{x}, 1 / 2+\mathrm{y}, 1 / 2-\mathrm{z})=2.88 \AA]$ are found at a distance shorter than the sum of the van der Waals radii and C9-H9...Cg1 $(-x+1, \quad y+1 / 2, \quad-z+1 / 2), \quad$ C9-H9...Cg3 $(-x+1$, $y+1 / 2,-z+1 / 2)$ interactions are present Table 2, where Cg1 and $\mathrm{Cg} 3$ are the centroids of the pyrone ring and the coumarin ring system, respectively. The resulting supramolecular aggregation is completed by the presence of $\pi-\pi$ stacking interactions between coumarin rings and between the $\mathrm{C} 4-\mathrm{C} 9$ benzene ring and pyrone or coumarin ring systems Figure 6. The centroid-centroid distances [Cg1...Cg2 (1-x, 2-y,1-z) = 3.6475(9), Cg2 ...Cg3 (1-x, 2$\mathrm{y}, 1-\mathrm{z})=3.6096(8), \mathrm{Cg} 3 \ldots \mathrm{Cg} 3(1-\mathrm{x}, 2-\mathrm{y}, 1-\mathrm{z})=3.6410(7) \AA$, where $\mathrm{Cg} 2$ is the centroid of the $\mathrm{C} 4-\mathrm{C} 9$ benzene ring] are less than $3.8 \AA$, the maximum regarded as suitable for an effective $\pi-\pi$ stacking interaction [19]. The perpendicular distances of $\mathrm{Cg}(\mathrm{I})$ on ring $\mathrm{J}$ and distances between $\mathrm{Cg}(\mathrm{I})$ and perpendicular projection of $\mathrm{Cg}(\mathrm{J})$ on ring I (slippage) [10] are summarized in Table 3.

Table 2. Hydrogen-bond geometry $\left(A,{ }^{\circ}\right)$.

\begin{tabular}{lllll}
\hline D-H...A & D-H & H...A & D...A & D-H...A \\
\hline $\mathrm{C} 8-\mathrm{H} 8 \ldots \mathrm{O} 4^{\mathrm{i}}$ & 0.93 & 2.47 & $3.341(2)$ & 156.0 \\
$\mathrm{C} 12-\mathrm{H} 12 \mathrm{~B} \ldots \mathrm{O} 2^{\text {ii }}$ & 0.96 & 2.59 & $3.511(3)$ & 160.6 \\
$\mathrm{C} 9-\mathrm{H} 9 \ldots \mathrm{Cg} 1^{\mathrm{iii}}$ & 0.93 & 2.47 & 2.95 & 120 \\
$\mathrm{C} 8-\mathrm{H} 8 \ldots \mathrm{Cg} 3^{\text {iii }}$ & 0.93 & 2.47 & 3.00 & 131 \\
\hline
\end{tabular}

Symmetry codes: (i) $x, y+1, z$; (ii) $x+1, y, z$; (iii) $-x+1, y+1 / 2,-z+1 / 2$

Table 3. Analysis of short ring interactions $(\AA)$.

\begin{tabular}{lllllll}
\hline $\mathbf{C g}(\mathbf{I})$ & $\mathbf{C g}(\mathbf{J})$ & Symmetry $\mathbf{C g}(\mathbf{J})$ & $\mathbf{C g}(\mathbf{I}) \ldots \mathbf{C g}(\mathbf{J})$ & CgI_Perp & CgJ_Perp & Slippage \\
\hline $\mathrm{Cg} 1$ & $\mathrm{Cg} 2$ & $1-\mathrm{x}, 2-\mathrm{y}, 1-\mathrm{z}$ & $3.6475(9)$ & $3.4499(6)$ & $3.4678(6)$ \\
$\mathrm{Cg} 2$ & $\mathrm{Cg} 1$ & $1-\mathrm{x}, 2-\mathrm{y}, 1-\mathrm{z}$ & $3.6475(9)$ & $3.4677(6)$ & $3.4500(6)$ \\
$\mathrm{Cg} 2$ & $\mathrm{Cg} 3$ & $1-\mathrm{x}, 2-\mathrm{y}, 1-\mathrm{z}$ & $3.6096(8)$ & $3.4544(6)$ & $3.4574(5)$ \\
$\mathrm{Cg} 3$ & $\mathrm{Cg} 2$ & $1-\mathrm{x}, 2-\mathrm{y}, 1-\mathrm{z}$ & $3.6097(8)$ & $3.4574(5)$ & $3.4545(6)$ & 1.037 \\
$\mathrm{Cg} 3$ & $\mathrm{Cg} 3$ & $1-\mathrm{x}, 2-\mathrm{y}, 1-\mathrm{z}$ & $3.6410(7)$ & $3.4537(5)$ & 1.047 & $3.4537(5)$ \\
\hline
\end{tabular}


Table 4. Fractional atomic coordinates and isotropic or equivalent isotropic displacement parameters $\left(\AA^{2}\right)$.

\begin{tabular}{|c|c|c|c|c|}
\hline Atom & $\mathbf{x}$ & $\mathbf{y}$ & $\mathbf{z}$ & $U_{\text {iso }} * / U_{\text {eq }}$ \\
\hline $\mathrm{O} 1$ & $0.415772(9)$ & $0.18169(18)$ & $0.06908(8)$ & $0.0680(3)$ \\
\hline $\mathrm{O} 3$ & $0.79324(10)$ & 0.3867 (2) & $0.10543(8)$ & $0.0780(4)$ \\
\hline $\mathrm{C} 5$ & $0.49827(13)$ & $0.3339(2)$ & $0.09983(9)$ & $0.0573(4)$ \\
\hline C6 & $0.60343(13)$ & $0.2770(2)$ & $0.09049(10)$ & $0.0610(4)$ \\
\hline H6 & 0.6175 & 0.1416 & 0.0645 & $0.073 *$ \\
\hline $\mathrm{O} 4$ & $0.81776(10)$ & $0.0941(9)$ & $0.20117(9)$ & 0.0827 (4) \\
\hline $\mathrm{C} 4$ & $0.47399(15)$ & $0.5364(2)$ & $0.13791(9)$ & $0.0638(4)$ \\
\hline $\mathrm{C} 7$ & $0.68714(14)$ & $0.4265(3)$ & $0.12073(10)$ & 0.0654 (4) \\
\hline $\mathrm{O} 2$ & $0.24121(12)$ & $0.0823(3)$ & $0.03833(13)$ & $0.1031(5)$ \\
\hline C9 & $0.56178(18)$ & $0.6816(3)$ & $0.16743(11)$ & $0.0776(5)$ \\
\hline H9 & 0.5483 & 0.8177 & 0.1931 & $0.093 *$ \\
\hline $\mathrm{C} 3$ & $0.36128(17)$ & $0.5800(3)$ & $0.14285(11)$ & $0.0762(5)$ \\
\hline H3 & 0.3429 & 0.7130 & 0.1684 & $0.091 *$ \\
\hline $\mathrm{C} 1$ & $0.30638(15)$ & $0.2246(3)$ & $0.07059(12)$ & 0.0747 (4) \\
\hline $\mathrm{C} 8$ & $0.66743(18)$ & $0.6290(3)$ & $0.15965(12)$ & $0.0788(5)$ \\
\hline H8 & 0.7252 & 0.7276 & 0.1802 & $0.095 *$ \\
\hline $\mathrm{C} 2$ & $0.28215(16)$ & $0.4320(3)$ & $0.11128(13)$ & $0.0794(5)$ \\
\hline $\mathrm{H} 2$ & 0.2095 & 0.4636 & 0.1157 & $0.095 *$ \\
\hline $\mathrm{C} 10$ & $0.85034(14)$ & $0.2070(3)$ & $0.14601(11)$ & 0.0697 (4) \\
\hline C11 & $0.95605(16)$ & $0.1802(4)$ & $0.11284(15)$ & $0.09332(6)$ \\
\hline H11A & 0.9396 & 0.1684 & 0.0461 & $0.112 *$ \\
\hline H11B & 1.0008 & 0.3125 & 0.1290 & $0.112 *$ \\
\hline $\mathrm{C} 12$ & $1.0203(2)$ & $-0.0149(7)$ & $0.1510(3)$ & $0.1554(15)$ \\
\hline $\mathrm{H} 12 \mathrm{~A}$ & 1.0346 & -0.0077 & 0.2172 & $0.233 *$ \\
\hline H12B & 1.0892 & -0.0171 & 0.1301 & $0.233^{*}$ \\
\hline $\mathrm{H} 12 \mathrm{C}$ & 0.9793 & -0.1477 & 0.1308 & $0.233 *$ \\
\hline
\end{tabular}

Table 5. Experimental and calculated bond lengths using $6-311^{++} G(d, p)$ basis set in $\AA$.

\begin{tabular}{|c|c|c|c|c|c|c|c|c|c|}
\hline Bond & X-Ray & DFT/RB3LYP & RMP2 & RHF & Bond & X-Ray & DFT/RB3LYP & RMP2 & RHF \\
\hline $\mathrm{O} 1-\mathrm{C} 5$ & $1.3766(18)$ & 1.364 & 1.366 & 1.349 & $\mathrm{C} 4-\mathrm{C} 3$ & $1.430(3)$ & 1.439 & 1.442 & 1.451 \\
\hline $\mathrm{O} 1-\mathrm{C} 1$ & $1.377(2)$ & 1.399 & 1.393 & 1.354 & $\mathrm{C} 7-\mathrm{C} 8$ & $1.387(2)$ & 1.399 & 1.401 & 1.389 \\
\hline $\mathrm{O} 3-\mathrm{C} 10$ & $1.361(2)$ & 1.380 & 1.382 & 1.347 & $\mathrm{O} 2-\mathrm{C} 1$ & $1.205(2)$ & 1.202 & 1.208 & 1.178 \\
\hline $\mathrm{O} 3-\mathrm{C} 7$ & $1.392(2)$ & 1.389 & 1.393 & 1.373 & $\mathrm{C} 8-\mathrm{C} 9$ & $1.367(3)$ & 1.382 & 1.391 & 1.374 \\
\hline $\mathrm{C} 5-\mathrm{C} 6$ & $1.374(2)$ & 1.392 & 1.397 & 1.385 & $\mathrm{C} 3-\mathrm{C} 2$ & $1.334(3)$ & 1.350 & 1.357 & 1.329 \\
\hline $\mathrm{C} 5-\mathrm{C} 4$ & $1.397(2)$ & 1.406 & 1.407 & 1.385 & $\mathrm{C} 1-\mathrm{C} 2$ & $1.440(3)$ & 1.458 & 1.464 & 1.468 \\
\hline $\mathrm{C} 6-\mathrm{C} 7$ & $1.374(2)$ & 1.387 & 1.390 & 1.374 & $\mathrm{C} 10-\mathrm{C} 11$ & $1.489(2)$ & 1.510 & 1.508 & 1.506 \\
\hline $\mathrm{O} 4-\mathrm{C} 10$ & $1.190(2)$ & 1.198 & 1.205 & 1.177 & $\mathrm{C} 11-\mathrm{C} 12$ & $1.463(4)$ & 1.526 & 1.525 & 1.523 \\
\hline $\mathrm{C} 4-\mathrm{C} 9$ & $1.391(3)$ & 1.409 & 1.409 & 1.395 & & & & & \\
\hline
\end{tabular}

Table 6. Experimental and calculated bond angles $\left({ }^{\circ}\right)$.

\begin{tabular}{|c|c|c|c|c|}
\hline Bond angle & X-Ray & DFT/RB3LYP/6-311 ${ }^{++} G(d, p)$ & RMP2/6-311 ${ }^{++} G(d, p)$ & RHF/6-311 ${ }^{++}$G(d,p) \\
\hline $\mathrm{C} 5-\mathrm{O} 1-\mathrm{C} 1$ & $122.18(13)$ & 122.90 & 122.21 & 123.70 \\
\hline $\mathrm{C} 10-\mathrm{O} 3-\mathrm{C} 7$ & $119.04(11)$ & 121.16 & 121.16 & 120.50 \\
\hline $\mathrm{C} 6-\mathrm{C} 5-\mathrm{O} 1$ & $116.90(13)$ & 116.98 & 116.10 & 117.15 \\
\hline $\mathrm{C} 6-\mathrm{C} 5-\mathrm{C} 4$ & $122.59(14)$ & 121.76 & 121.46 & 121.51 \\
\hline $\mathrm{O} 1-\mathrm{C} 5-\mathrm{C} 4$ & $120.51(14)$ & 121.26 & 121.97 & 121.34 \\
\hline $\mathrm{C} 5-\mathrm{C} 6-\mathrm{C} 7$ & $117.89(14)$ & 118.14 & 118.23 & 118.35 \\
\hline $\mathrm{C} 9-\mathrm{C} 4-\mathrm{C} 5$ & $117.16(16)$ & 118.28 & 118.58 & 118.52 \\
\hline $\mathrm{C} 9-\mathrm{C} 4-\mathrm{C} 3$ & $124.97(15)$ & 124.26 & 123.87 & 124.28 \\
\hline $\mathrm{C} 5-\mathrm{C} 4-\mathrm{C} 3$ & $117.87(15)$ & 117.47 & 117.45 & 117.20 \\
\hline $\mathrm{C} 6-\mathrm{C} 7-\mathrm{C} 8$ & $121.72(16)$ & 121.73 & 121.95 & 121.85 \\
\hline $\mathrm{C} 6-\mathrm{C} 7-\mathrm{O} 3$ & $120.31(14)$ & 121.98 & 120.07 & 120.56 \\
\hline $\mathrm{C} 8-\mathrm{C} 7-\mathrm{O} 3$ & $117.73(14)$ & 116.16 & 117.85 & 117.50 \\
\hline $\mathrm{C} 8-\mathrm{C} 9-\mathrm{C} 4$ & $117.87(15)$ & 120.82 & 120.67 & 121.02 \\
\hline $\mathrm{C} 2-\mathrm{C} 3-\mathrm{C} 4$ & $121.72(16)$ & 120.84 & 120.16 & 120.47 \\
\hline $\mathrm{O} 2-\mathrm{C} 1-\mathrm{O} 1$ & $120.31(14)$ & 117.71 & 118.09 & 118.74 \\
\hline $\mathrm{O} 2-\mathrm{C} 1-\mathrm{C} 2$ & $126.98(18)$ & 126.41 & 125.61 & 124.92 \\
\hline $\mathrm{O} 1-\mathrm{C} 1-\mathrm{C} 2$ & $116.96(16)$ & 115.88 & 116.31 & 116.33 \\
\hline $\mathrm{C} 9-\mathrm{C} 8-\mathrm{C} 7$ & $119.03(16)$ & 119.26 & 119.02 & 118.75 \\
\hline $\mathrm{C} 3-\mathrm{C} 2-\mathrm{C} 1$ & $121.64(17)$ & 121.65 & 121.85 & 120.96 \\
\hline $\mathrm{O} 4-\mathrm{C} 10-\mathrm{O} 3$ & $122.59(16)$ & 123.63 & 123.35 & 123.26 \\
\hline $\mathrm{O} 4-\mathrm{C} 10-\mathrm{C} 11$ & $126.85(18)$ & 126.88 & 127.04 & 126.51 \\
\hline $\mathrm{O} 3-\mathrm{C} 10-\mathrm{C} 11$ & $110.55(14)$ & 109.49 & 109.61 & 110.23 \\
\hline $\mathrm{C} 12-\mathrm{C} 11-\mathrm{C} 10$ & $113.71(19)$ & 113.20 & 112.30 & 113.24 \\
\hline
\end{tabular}


Table 7. Experimental and calculated torsion angles $\left({ }^{\circ}\right)$.

\begin{tabular}{|c|c|c|c|c|}
\hline Torsion angle & X-ray & DFT/RB3LYP/6-311 ${ }^{++}$G(d,p) & RMP2/6-311 ${ }^{++} G(d, p)$ & RHF/6-311 ${ }^{++}$G(d,p) \\
\hline $\mathrm{C} 1-\mathrm{O} 1-\mathrm{C} 5-\mathrm{C} 6$ & $-176.72(13)$ & 179.76 & 179.86 & 179.97 \\
\hline $\mathrm{C} 1-\mathrm{O} 1-\mathrm{C} 5-\mathrm{C} 4$ & $2.8(2)$ & -0.04 & -2.15 & -0.18 \\
\hline $\mathrm{O} 1-\mathrm{C} 5-\mathrm{C} 6-\mathrm{C} 7$ & $179.99(13)$ & 179.94 & -179.40 & 179.87 \\
\hline $\mathrm{C} 4-\mathrm{C} 5-\mathrm{C} 6-\mathrm{C} 7$ & $0.4(2)$ & 0.23 & 2.60 & -0.04 \\
\hline $\mathrm{C} 6-\mathrm{C} 5-\mathrm{C} 4-\mathrm{C} 9$ & $-0.5(2)$ & 0.03 & -2.75 & 0.15 \\
\hline $\mathrm{O} 1-\mathrm{C} 5-\mathrm{C} 4-\mathrm{C} 9$ & $180.00(13)$ & -179.67 & 179.36 & -179.76 \\
\hline $\mathrm{C} 6-\mathrm{C} 5-\mathrm{C} 4-\mathrm{C} 3$ & $178.95(13)$ & -179.99 & -179.38 & -179.90 \\
\hline $\mathrm{O} 1-\mathrm{C} 5-\mathrm{C} 4-\mathrm{C} 3$ & $-0.6(2)$ & 0.31 & 2.73 & 0.19 \\
\hline $\mathrm{C} 5-\mathrm{C} 6-\mathrm{C} 7-\mathrm{C} 8$ & $0.0(2)$ & -0.40 & -2.08 & -0.24 \\
\hline $\mathrm{C} 5-\mathrm{C} 6-\mathrm{C} 7-\mathrm{O} 3$ & $-174.16(13)$ & -176.26 & -177.88 & -176.70 \\
\hline $\mathrm{C} 10-\mathrm{O} 3-\mathrm{C} 7-\mathrm{C} 6$ & $-64.08(19)$ & -51.59 & -69.38 & -114.31 \\
\hline $\mathrm{C} 10-\mathrm{O} 3-\mathrm{C} 7-\mathrm{C} 8$ & $121.49(16)$ & 132.32 & 114.65 & 69.19 \\
\hline $\mathrm{C} 5-\mathrm{C} 4-\mathrm{C} 9-\mathrm{C} 8$ & $0.0(2)$ & -179.75 & 2.36 & -0.24 \\
\hline $\mathrm{C} 3-\mathrm{C} 4-\mathrm{C} 9-\mathrm{C} 8$ & $-179.38(15)$ & 179.88 & 178.76 & -179.93 \\
\hline $\mathrm{C} 9-\mathrm{C} 4-\mathrm{C} 3-\mathrm{C} 2$ & $178.82(16)$ & 179.74 & -178.60 & 179.82 \\
\hline $\mathrm{C} 5-\mathrm{C} 4-\mathrm{C} 3-\mathrm{C} 2$ & $-0.5(2)$ & -0.24 & -2.17 & -0.13 \\
\hline $\mathrm{C} 5-\mathrm{O} 1-\mathrm{C} 1-\mathrm{O} 2$ & $177.03(16)$ & 179.67 & -179.31 & 179.94 \\
\hline $\mathrm{C} 5-\mathrm{O} 1-\mathrm{C} 1-\mathrm{C} 2$ & $-3.7(2)$ & -0.28 & 0.87 & -0.06 \\
\hline $\mathrm{C} 4-\mathrm{C} 9-\mathrm{C} 8-\mathrm{C} 7$ & $0.5(2)$ & -0.01 & -1.86 & -0.28 \\
\hline $\mathrm{C} 6-\mathrm{C} 7-\mathrm{C} 8-\mathrm{C} 9$ & $-0.5(2)$ & 0.28 & 1.75 & 0.40 \\
\hline $\mathrm{O} 3-\mathrm{C} 7-\mathrm{C} 8-\mathrm{C} 9$ & $173.85(14)$ & 176.38 & 177.63 & 176.75 \\
\hline $\mathrm{C} 4-\mathrm{C} 3-\mathrm{C} 2-\mathrm{C} 1$ & $-0.5(3)$ & -0.08 & 1.04 & -0.01 \\
\hline $\mathrm{O} 2-\mathrm{C} 1-\mathrm{C} 2-\mathrm{C} 3$ & $-178.29(19)$ & -179.63 & 179.85 & -179.87 \\
\hline $\mathrm{O} 1-\mathrm{C} 1-\mathrm{C} 2-\mathrm{C} 3$ & $2.6(3)$ & 0.33 & -0.34 & -0.09 \\
\hline $\mathrm{C} 7-\mathrm{O} 3-\mathrm{C} 10-\mathrm{O} 4$ & $-7.4(2)$ & -1.56 & -1.59 & -0.65 \\
\hline $\mathrm{C} 7-\mathrm{O} 3-\mathrm{C} 10-\mathrm{C} 11$ & $173.74(15)$ & 178.78 & 178.56 & 179.22 \\
\hline $\mathrm{O} 4-\mathrm{C} 10-\mathrm{C} 11-\mathrm{C} 12$ & $3.3(3)$ & -0.62 & -2.81 & -0.79 \\
\hline $\mathrm{O} 3-\mathrm{C} 10-\mathrm{C} 11-\mathrm{C} 12$ & $-177.9(2)$ & 179.03 & 177.04 & 179.34 \\
\hline
\end{tabular}

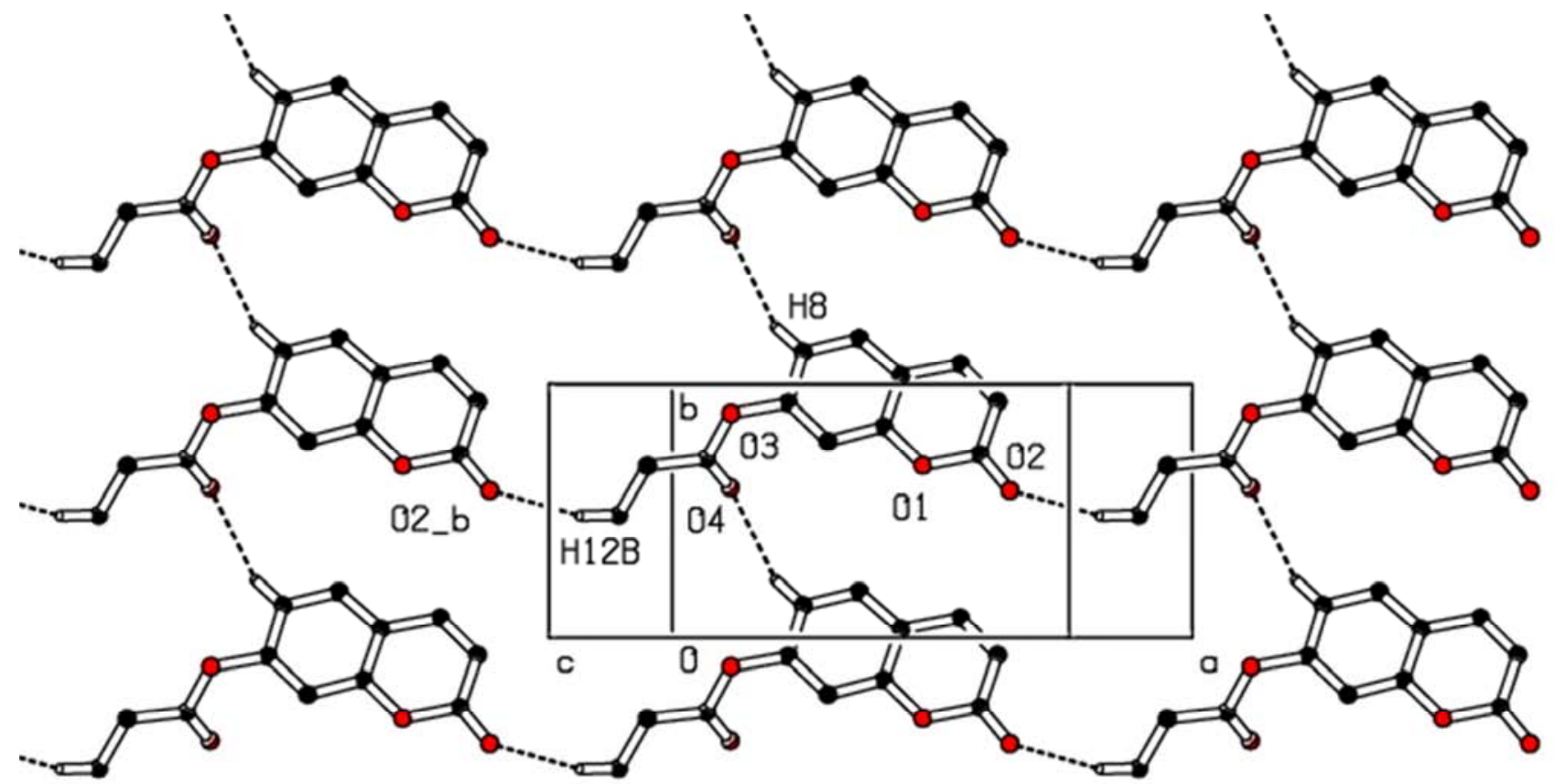

Figure 5. Part of the crystal packing of the title compound showing the formation of an infinite $R_{4}^{4}(30)$ motifs in the ab plane. Dashed lines indicate hydrogen bonds. H atoms not involved in hydrogen bonding have been omitted for clarity. 


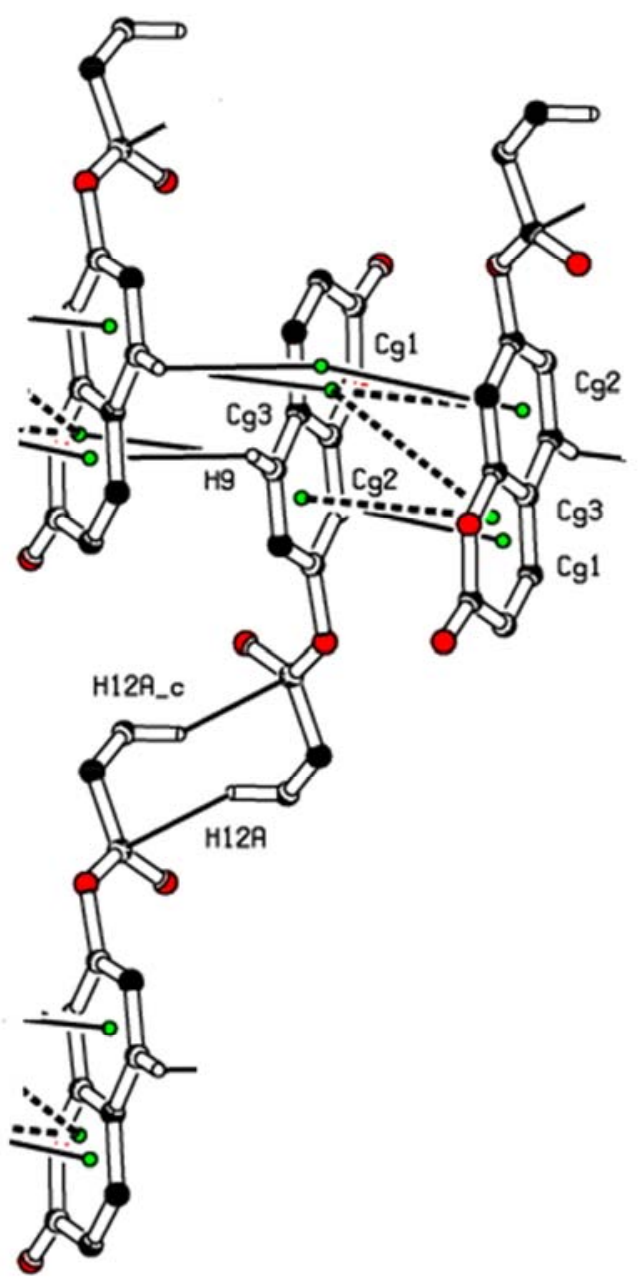

Figure 6. A view of the crystal packing, showing $H \ldots . . C$ contacts, $C-H \ldots \pi$ and $\pi-\pi$ stacking interactions (dashed lines). The green dots are ring centroids. $H$ atoms not involved in H...C or H... $\pi$ interactions have been omitted for clarity.

\section{Hirshfeld Surface Analysis}

The analysis of intermolecular interactions through the mapping of three-dimensional $\mathrm{d}_{\text {norm }}$ involves the contact

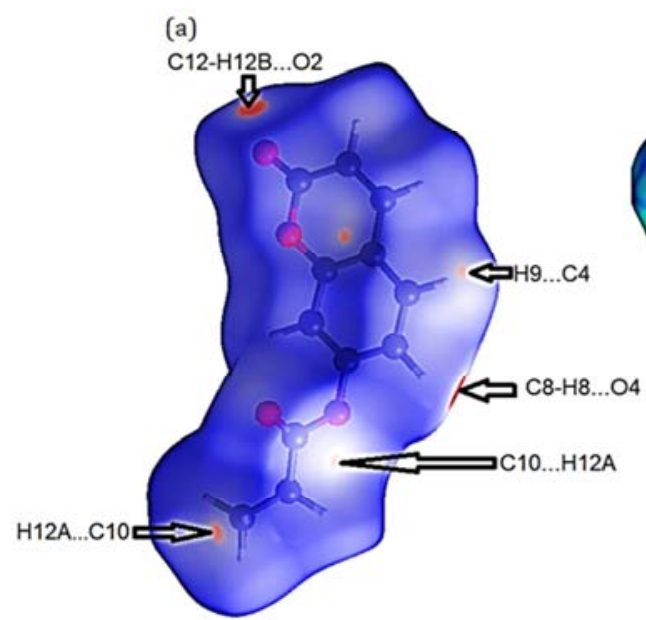

distances $d_{i}$ and $d_{e}$ from the Hirshfeld surface to the nearest atom inside and outside, respectively. In the studied coumarin, the surface mapped over $d_{\text {norm }}$ highlights two brite red and three faint-red spots, reflecting distances shorter than the sum of the van der Waals radii. These dominant interactions correspond to intermolecular $\mathrm{C}-\mathrm{H}$... hydrogen bonds, H...C, C-H... and $\pi-\pi$ stacking interactions between the surface and the neighbouring environment. The mapping also shows white spots, with distances equal to the sum of the van der Waals radii, and blue regions with distances longer than the sum of the van der Waals radii.

Transparent surfaces are displayed in order to visualize the molecule Figure $7 \mathrm{a}$. In the shape-index map ( -1.00 to 1.00 a.u., Figure $7 \mathrm{~b}$ ), the adjacent red and blue triangle-like patches show concave regions that indicate $\pi-\pi$ stacking interactions [20]. Furthermore, the two-dimensional fingerprint plots (FP) are decomposed to highlight particular close contacts of atom pairs, and the contributions from different contacts are provided in Figure 8 . The blue spots in the middle of the surface appearing near $\mathrm{d}_{\mathrm{e}}=\mathrm{d}_{\mathrm{i}} \simeq 1.8-2.0 \AA$ correspond to close C...C interplanar contacts. These contacts, which comprise $4.8 \%$ of the total Hirshfeld surface area, are related to $\pi-\pi$ interactions Figure $8 \mathrm{a}$ as predicted by the X-ray study. The most significant contribution to the Hirshfeld surface $(38.1 \%)$ is from $\mathrm{H} \ldots \mathrm{O} / \mathrm{O} \ldots \mathrm{H}$ contacts, which appear on the left side as blue spikes with the tip at $d_{e}$ $+\mathrm{d}_{\mathrm{i}} \simeq 2.6 \AA$, top and bottom Figure 8b, showing the presence of $\mathrm{O} \ldots \mathrm{H}$ contacts. As expected in organic compounds, the H...H contacts are important with a $35.3 \%$ contribution to Hirshfeld surface; these appear in the central region of the FP with a central blue tip spike at $d_{e}=d_{i}=1.30$ $\AA$ Figure $8 \mathrm{c}$ whereas the C...H/H...C plot (15.9\%) reveals information on the intermolecular hydrogen bonds Figure $8 \mathrm{~d}$. Other visible spots in the Hirshfeld surfaces showing C...O/O...C and O...O contacts make contributions for 5.0 and $0.9 \%$, respectively Figures $8 \mathrm{e}$ and $8 \mathrm{f}$.

Figure 7. Hirshfeld surfaces mapped over $d_{\text {norm }}(-0.205$ to 1.418 a.u.) (left) and shape-index (right). 
(a)

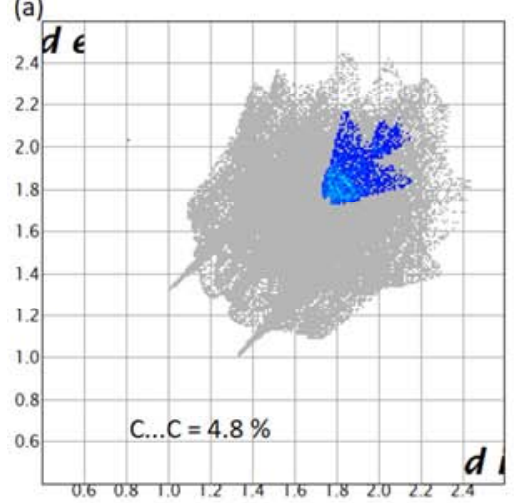

(d)

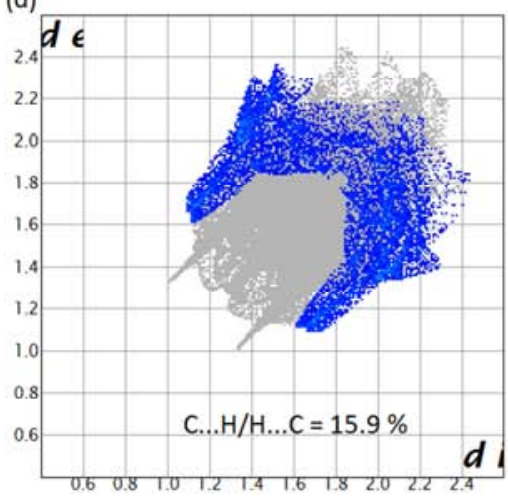

(b)

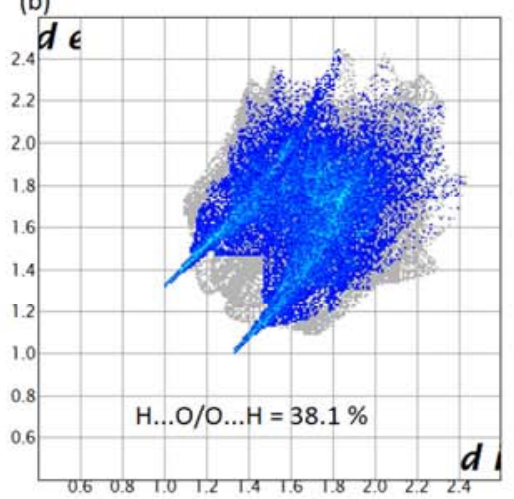

(e)

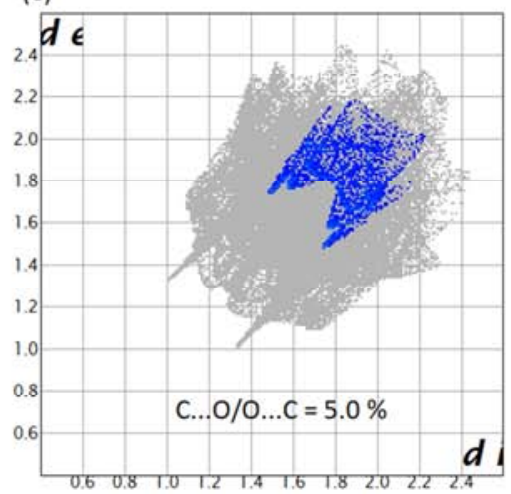

(c)

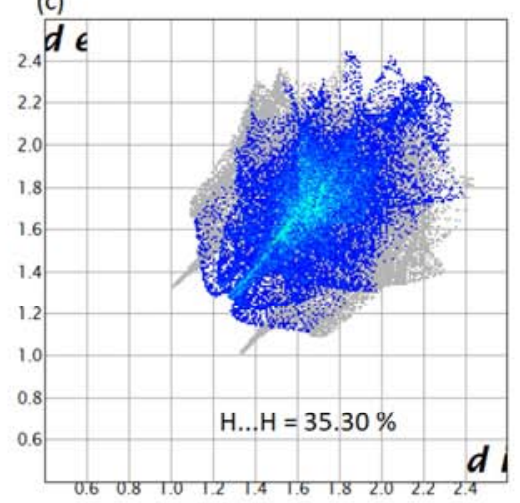

(f)

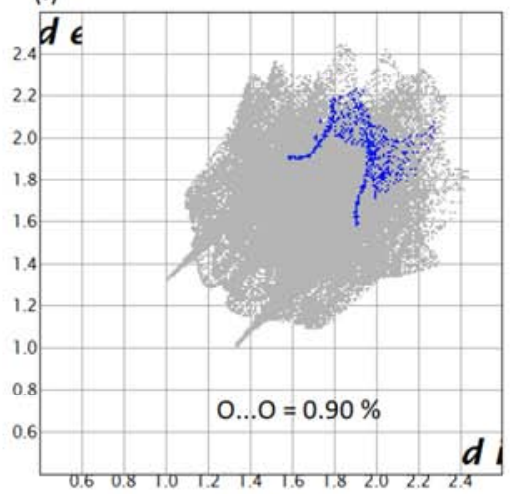

Various close contacts and their relative contributions are indicated.

Figure 8. Decomposed two-dimensional fingerprint plots for the title compound.

\section{Theoretical Calculations}

\subsection{Quantum Chemical Computational Studies}

The resulting geometrical parameters are compared with those obtained from the X-ray crystallographic study. An analysis of the computational bond lengths and bond angles and comparison with the crystallographic results shows a good agreement between them, with a root-mean-square deviation of $0.021 \AA$ (DFT/RB3LYP), $0.022 \AA$ (RMP2) and $0.022 \AA$ (RHF) for bond lengths and $1.21^{\circ}$ (DFT/RB3LYP), $1.20^{\circ}$ (RMP2) and $1.13^{\circ}$ (RHF) for bond angles Tables 5 and 6. In addition, an inspection of the calculated torsion angles shows that the coumarin ring system is planar, which is in good agreement with the crystallographic prevision, although the observed $\mathrm{C} 10-\mathrm{O} 3-\mathrm{C} 7-\mathrm{C} 8$ torsion angle between this ring system and the 7-propionate side chain $\left(121.49(16)^{\circ}\right)$ is somewhat lower than the DFT/RB3LYP calculated value [132.32 ${ }^{\circ}$ and larger than the RMP2 [114.65] and the RHF [69.19] values Table 7.

\subsection{Molecular Electrostatic Potential (MEP)}

The molecular electrostatic potential surface and contour map may lead to better understanding sites for electrophilic attack and nucleophilic reactions as well as hydrogenbonding interactions [21-23] of the compound under study. The molecular electrostatic potential, $(r)$, may be either positive or negative in any given region, depending upon whether the effect of the nuclei or the electrons is dominant there.

For convenience, $\mathrm{V}(\mathrm{r})$ is typically written in terms of atomic units, a.u; it then has the following form [24]:

$$
V(r)=\sum_{A} \frac{z_{A}}{\left|R_{A}-r\right|}-\int \frac{\rho\left(r^{\prime}\right)}{\left|r^{\prime}-r\right|} d^{3} r^{\prime}
$$

where $Z_{A}$ is the charge of nucleus A located at $R_{A}, \rho\left(r^{\prime}\right)$ is the electronic density function of the molecule, and $r$ ' is the dummy integration variable. Being a real physical property, $\mathrm{V}(r)$ can be determined experimentally by diffraction or by computational methods [25]. To predict reactive sites for electrophilic and nucleophilic attack for the title molecule, MEP was computed at the DFT/RB3LYP, RMP2 and RHF optimized geometries using the $6-311^{++} \mathrm{G}(\mathrm{d}, \mathrm{p})$ basis set. The negative (red) regions of MEP were related to electrophilic reactivity and the positive (blue) regions to nucleophilic reactivity shown in Figure 9. As can be seen from the figure, there are two possible sites on compound (I) for electrophilic attack. These negative regions are localised on the oxygen atoms $\mathrm{O} 2$ and $\mathrm{O} 4$ with a maximum value of $-0.095,-0.078$ and -0.079 a.u. for DFT/RB3LYP/6-311 ${ }^{++} \mathrm{G}(\mathrm{d}, \mathrm{p})$, RMP2/6$311^{++} \mathrm{G}(\mathrm{d}, \mathrm{p})$ and $\mathrm{RHF} / 6-311^{++} \mathrm{G}(\mathrm{d}, \mathrm{p})$ basis sets, respectively. These results provide information concerning the region where the studied compound can interact intermolecularly. Therefore, Figure 9 confirms the existence of the intermolecular $\mathrm{C} 12-\mathrm{H} 12 \mathrm{~B} . . . \mathrm{O} 2$ interaction. 


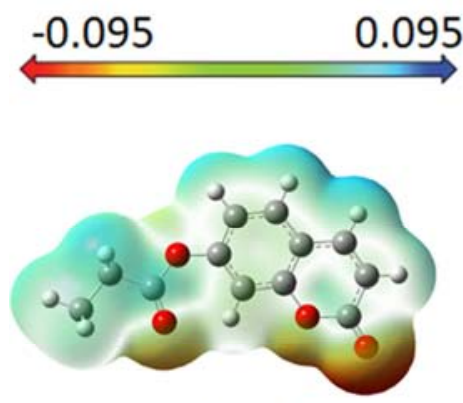

DFT/RB3LYP/6-311 ${ }^{+} \mathrm{G}(\mathrm{d}, \mathrm{p})$

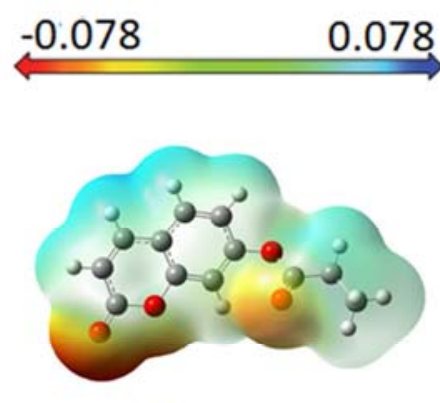

RMP2/6-311++G(d,p)
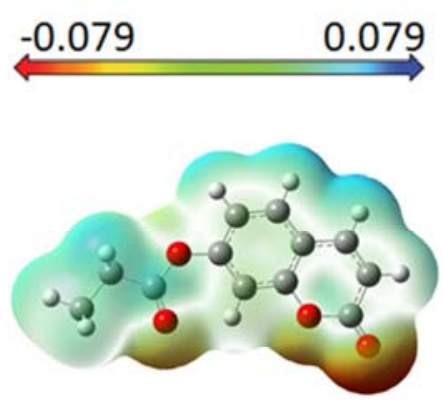

RHF/6-311++G(d,p)

Figure 9. Molecular electrostatic potential map (MEP) (in a..u.) calculated at DFT/RB3LYP/6-311 ${ }^{++} G(d, p), R M P 2 / 6-311^{++} G(d, p)$ and $R H F / 6-311^{++} G(d, p)$ level frontier molecular orbitals analysis.
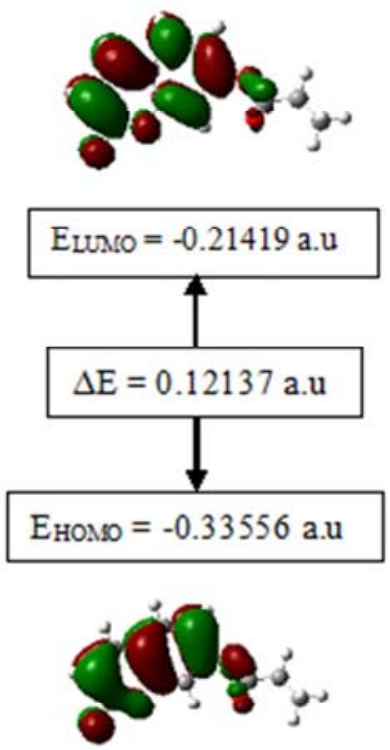

DFT/RB3L YP/6-311+ G(d,p)
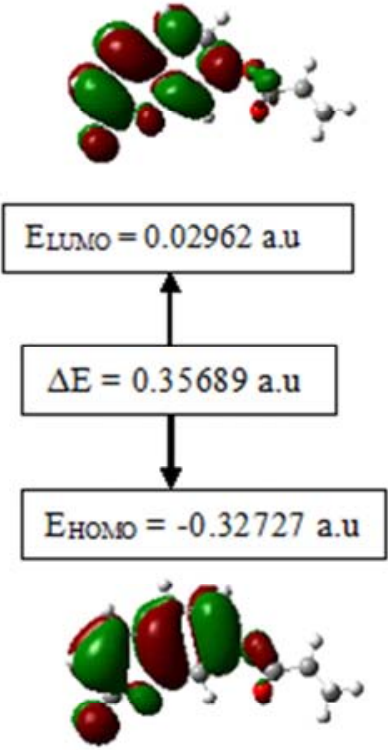

$\operatorname{RMP} 2 / 6-311^{++} \mathrm{G}(\mathrm{d}, \mathrm{p})$
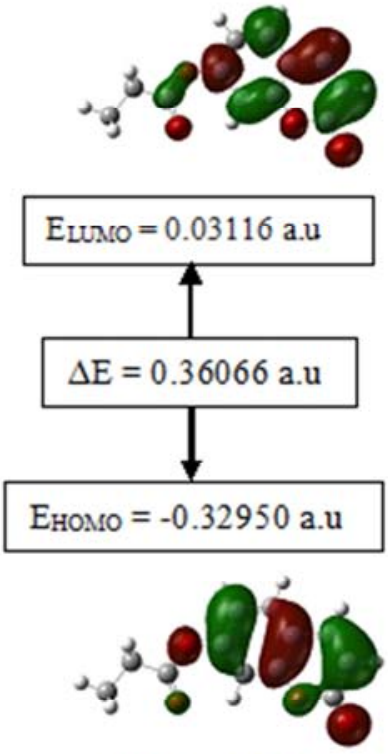

RHF/6-311 ${ }^{+} \mathrm{G}(\mathrm{d}, \mathrm{p})$

Figure 10. The distributions and energy levels of the HOMO and LUMO orbitals computed at the DFT/RB3LYP/6-311 ${ }^{++} G(d, p), R M P 2 / 6-311^{++} G(d, p)$ and $R H F / 6-311^{++} G(d, p)$ levels for the title compound.

Table 8. Mulliken atomic charges computed with $6-311^{++} G(d, p)$ basis set.

\begin{tabular}{lllllll}
\hline Atom & DFT/RB3LYP & RMP2 & RHF & Atom & DFT/RB3LYP & RMP2 \\
\hline O1 & -0.112 & -0.253 & -0.260 & RHF & -0.668 & -0.983 \\
O2 & -0.275 & -0.364 & -0.363 & C8 & 0.401 & 0.381 \\
O3 & -0.011 & -0.048 & -0.046 & H8 & 0.197 & 0.151 \\
O4 & -0.192 & -0.231 & -0.249 & C9 & 0.027 & 0.239 \\
C1 & 0.202 & 0.315 & 0.348 & H9 & 0.153 & -0.010 \\
C2 & -0.050 & -0.078 & -0.077 & C10 & -0.185 & -0.203 \\
H2 & 0.220 & 0.271 & 0.262 & C11 & -0.083 & -0.983 \\
C3 & -0.257 & -0.314 & -0.280 & H11A & 0.180 & -0.046 \\
H3 & 0.167 & 0.221 & 0.208 & H11B & 0.175 & 0.179 \\
C4 & 2.351 & 2.243 & 2.254 & C12 & -0.595 & 0.178 \\
C5 & -1.950 & -1.541 & -2.153 & H12A & 0.162 & -0.161 \\
C6 & -0.404 & -0.269 & 0.024 & H12B & 0.166 & 0.176 \\
H6 & 0.245 & 0.283 & 0.271 & H12C & 0.139 & 0.164 \\
\hline
\end{tabular}

\subsection{HOMO-LUMO Analysis}

The distributions and energy levels of the highest occupied molecular orbital (HOMO) and the lowest lying unoccupied molecular orbital (LUMO) calculated at the DFT/RB3LYP/6$311^{++} \mathrm{G}(\mathrm{d}, \mathrm{p}), \quad \mathrm{RMP} 2 / 6-311^{++} \mathrm{G}(\mathrm{d}, \mathrm{p})$ and $\mathrm{RHF} / 6-311^{++} \mathrm{G}(\mathrm{d}, \mathrm{p})$ level for the title compound are shown in Figure 10. The calculations indicate that the title compound has 57 occupied molecular orbitals and the value of the energy separation between the LUMO and HOMO are 0.12137, 0.35689 and 0.36066 a.u for at the same levels, respectively. These frontier 
orbital gaps in the range 0.12137-0.3606 a.u show that 2-oxo$2 \mathrm{H}$-chromen-7-yl propionate is polarizable and is associated with a high chemical reactivity and low kinetic stability and is also termed as soft molecule [26]. The HOMO and LUMO energies, the energy gap $(\Delta E)$, the ionization potential $(I)$, the electron affinity $(A)$, the absolute electronegativity $(\chi)$, the absolute hardness $(\eta)$, and softness $(S)$ for compound (I) have been computed at the same levels and the results are given in Table 9. By using HOMO and LUMO energy values for a molecule, electronegativity and chemical hardness can be calculated as follows [27]:

$$
\begin{gathered}
\chi=-\left(\mathrm{E}_{\text {Hомо }}+\mathrm{E}_{\text {Lumo }}\right) / 2 \\
\eta=\left(\mathrm{E}_{\text {LUмо }}-\mathrm{E}_{\text {Hомо }}\right) / 2 \\
S=1 / 2 \eta \\
I=-E_{\text {Hомо }} \\
A=-E_{\text {LUMO }}
\end{gathered}
$$

\begin{tabular}{|c|c|c|c|}
\hline & DFT/R B3LYP & RMP2 & RHF \\
\hline $\mathrm{E}_{\text {LUMO }}$ (a.u) & -0.21419 & 0.02962 & 0.03116 \\
\hline $\mathrm{E}_{\text {Номо }}(\mathrm{a} . \mathrm{u})$ & -0.33556 & -0.32727 & -0.32950 \\
\hline I (a.u) & 0.33556 & 0.32727 & 0.32950 \\
\hline A (a.u) & 0.21419 & -0.02962 & -0.03116 \\
\hline$\chi($ a.u) & 0.274875 & 0.148825 & 0.14917 \\
\hline$\eta$ (a.u) & 0.060685 & 0.178445 & 0.18033 \\
\hline$S\left(\right.$ a. $\left.^{-1}\right)$ & 8.23927 & 2.801984 & 2.77269 \\
\hline
\end{tabular}

Table 9. The calculated frontier orbital energies, electronegativity, hardness and softness of the title compound using DFT/RB3LYP/6-311 ${ }^{++} G(d, p)$, $R M P 2 / 6-311^{++} G(d, p)$ and $R H F / 6-311^{++} G(d, p)$ levels.

\subsection{The Mulliken Charge Population}

The Mulliken atomic charge calculation has an important role in the application of quantum chemical calculation to molecular system because atomic charges effect dipole moment, molecular polarizability, electronic structure, and a lot of properties of molecular systems. The charge distributions calculated by the Mulliken method [28-31] for the equilibrium geometry of the title compound is given in Table 8. The computed Mulliken charges of $\mathrm{C} 12$ and $\mathrm{H} 12 \mathrm{~B}$ atoms are determined as -0.595 and $0.166 \mathrm{e},-0.651$ and 0.165 e, -0.652 and 0.164 e for the DFT/RB3LYP $/ 6-311^{++} \mathrm{G}(\mathrm{d}, \mathrm{p})$, RMP $2 / 6-311^{++} \mathrm{G}(\mathrm{d}, \mathrm{p})$ and $\mathrm{RHF} / 6-311^{++} \mathrm{G}(\mathrm{d}, \mathrm{p})$ methods, respectively. These values confirm intermolecular hydrogen bond $\mathrm{C} 12-\mathrm{H} 12 \mathrm{~B} \ldots \mathrm{O} 2[\mathrm{x}+1, \mathrm{y}, \mathrm{z}]$. However, the $\mathrm{C} 8-$ $\mathrm{H} 8 \ldots \mathrm{O} 4[\mathrm{x}, \mathrm{y}+1, \mathrm{z}]$ obseved in the solid state is not discernable in the gas phase. Also, the calculated Mulliken charges of $\mathrm{C} 3$ and H3, C6 and H6 atoms Table 8 may suggest other intermolecular contacts in the gaseous state.

\section{Conclusions}

In this present investigation, molecular structure was analyzed by X-ray cristallography and the intermolecular interactions by Hirshfeld surface analysis. Also, molecular electrostatic potential, HOMO-LUMO analysis and the
Mulliken charge populations of 2-oxo-2H-chromen-7-yl propionate have been studied using DFT/RB3LYP/6$311^{++} \mathrm{G}(\mathrm{d}, \mathrm{p}), \quad \mathrm{RMP} 2 / 6-311^{++} \mathrm{G}(\mathrm{d}, \mathrm{p})$ and $\mathrm{RHF} / 6-311^{++} \mathrm{G}(\mathrm{d}, \mathrm{p})$ calculations. The calculated geometric parameters (bond lenght, bond angle, and torsion angle) are compared with their experimental data. It is seen that there are no significant differences, when the experimental structure is compared with theoretical structures except the experimental torsion angle, $\mathrm{C} 10-\mathrm{O} 3-\mathrm{C} 7-\mathrm{C} 8$, which differs from those of the calculeted values. The MEP maps show that the negative potential sites are on electronegative atoms and the positive potential sites are around the hydrogen atoms. These sites provide information concerning the region from where the compound can undergo intra- and intermolecular interactions. Similarly, the Mulliken charges confirm the intermolecular C12-H12B...O2 hydrogen bond in the solid state.

\section{Acknowledgements}

The Spectropole Service of the Federation of Chemical Sciences (Aix-Marseille University, France) is gratefully acknowledged for its help with the whole analysis.

\section{Conflict of Interests}

All the authors do not have any possible conflicts of interest.

\section{References}

[1] Basanagouda M, Kulkarni M V, Sharma D, Gupta V K, Pranesha P, Sandhyarani P and Rasal V P, J. Chem. Sci. (2009); 121: 485-495.

[2] Vuković N, Sukdolak S, Solujić S and Niciforović N, Arch. Pharm. Res. (2010); 33: 5-15.

[3] Emmanuel-Giota A A, Fylaktakidou K C, Litinas K E, Nicolaides D N and Hadjipavlou-Litina D J, Heterocycl. Chem. (2001); 38: 717-722.

[4] Abou A, Djandé A, Kakou-Yao R, Saba A and Tenon A J, Acta Cryst. (2013); E69: o1081-o1082.

[5] Ouédraogo M, Abou A, Djandé A, Ouari O and Zoueu T J, Acta Cryst. (2018); E74: 530-534.

[6] Rigaku OD (2015). CrysAlis PRO. Rigaku Oxford Diffraction, Yarnton, England.

[7] Burla M C, Caliandro R, Carrozzini B, Cascarano G L, Cuocci C, Giacovazzo C, Mallamo M, Mazzone A and Polidori G, J. Appl. Cryst. (2015), 48: 306-309.

[8] Farrugia L J, J. Appl. Cryst. (2012); 45: 849-854.

[9] Sheldrick G M, Acta Cryst. (2015); C71: 3-8.

[10] Spek A L, Acta Cryst. (2009); D65: 148-155.

[11] Wolff S K, Grimwood D J, McKinnon J J, Turner M J, Jayatilaka D and Spackman M A (2012). Crystal Explorer. The University of Western Australia. 
[12] Frisch M J, Trucks G W, Schlegel H B, Scuseria G E, Robb M A, Cheeseman J R, et al. (2013). GAUSSIAN09. Gaussian, Inc., Wallingford, CT, USA.

[13] Allen F H, Walson D G, Orpen A G, Taylor R, Tables of Bonds Lengths by X-Ray and Neutron diffraction. Part 1. Bonds Lenths in organic compounds. J. Chem. Soc., Perkin Trans. II (1987), S1-S9.

[14] Abou A, Yoda J, Djandé A, Coussan S and Zoueu T J, Acta Cryst. (2018); E74: 761-765.

[15] Djandé A, Abou A, Kini F, Kambo K R and Giorgi M, IUCrData (2018); 3: x180927.

[16] Gomes L R, Low J N, Fonseca A, Matos M J and Borges F, Acta Cryst. (2016); E72: 926-932.

[17] Ziki E, Yoda J, Djandé A, Saba A and Kakou-Yao R, Acta Cryst. (2016); E72: 1562-1564.

[18] Bernstein J, Davis R E, Shimoni L and Chang N-L, Angew. Chem. Int. Ed. Engl. (1995); 34: 1555-1573.

[19] Janiak J, J. Chem. Soc. Dalton Trans. (2000), 3885-3896.

[20] Bitzer S R, Visentin C L, Hörner M, Nascimento M A C and Filgueiras C A L, J. Mol. Struct. (2017); 1130: 165-173.

[21] E. Scrocco and J. Tomasi, "Electronic molecular structure, reactivity and intermolecular forces: an euristic interpretation by means of electrostatic molecular potentials," Advances in Quantum Chemistry, 1979; vol. 11, pp. 115-193, 1979.

[22] F. J. Luque, J. M. López, and M. Orozco, "Perspective on "Electrostatic interactions of a solute with a continuum. A direct utilization of ab initio molecular potentials for the prevision of solvent effects", Theoretical Chemistry Accounts, vol. 103, no. 3-4, pp. 343-345, 2000.
[23] N. Okulik and A. H. Jubert, "Theoretical analysis of the reactive sites of non-steroidal anti-inflammatory drugs," Internet Electronic Journal of Molecular Design, vol. 4, pp. 17-30, 2005.

[24] P. Politzer and J. S. Murray, "The fundamental nature and role of the electrostatic potential in atoms and molecules," Theoretical Chemistry Accounts, vol. 108, no. 3, pp. 134-142, 2002.

[25] P. Politzer and D. G. Truhlar, Chemical Applications of Atomic and Molecular Electrostatic Potentials, Plenum, New York, NY, USA, 1981.

[26] I. Fleming, Frontier Orbitals and Organic Chemical Reactions, John Wiley \& Sons, London, UK, 1976.

[27] R. G. Pearson, "Absolute electronegativity and hardness correlated with molecular orbital theory," Proceedings of the National Academy of Sciences of the United States of America, vol. 83, no. 22, pp. 8440-8841, 1986.

[28] R. S. Mulliken, "Electronic population analysis on LCAOMO molecular wave functions. I," The Journal of Chemical Physics, vol. 23, no. 10, pp. 1833-1840, 1955.

[29] R. S. Mulliken, "Electronic population analysis on LCAOMO molecular wave functions. II. Overlap populations, bond orders, and covalent bond energies," The Journal of Chemical Physics, vol. 23, no. 10, pp. 1841-1846, 1955.

[30] R. S. Mulliken, "Electronic population analysis on LCAOMO molecular wave functions. III. effects of hybridization on overlap and gross AO populations," The Journal of Chemical Physics, vol. 23, no. 12, pp. 2338-2342, 1955.

[31] R. S. Mulliken, "Electronic population analysis on LCAOMO molecular wave functions. IV. bonding and antibonding in LCAO and valence-bond theories," The Journal of Chemical Physics, vol. 23, no. 12, pp. 2343-2346, 1955. 